Adequate glucose control can only be achieved with repetitive blood sampling which means mainly repetitive pain- and stressful procedures many times daily.

Devices measuring glucose levels subcutaneously (CGMS) are widely used in adults and children suffering from diabetes. The feasibility and accuracy of such devices were never evaluated in newborn infants.

Methods In this pilot study CGMS sensors were placed in infants (GA $>35+0$, birthweight $>2500 \mathrm{~g}$ ) at risk of hypoglycaemia in addition to routine glucose sampling (RG). The CGMS generates a glucose value every 5 minutes and needs calibration twice a day. Because of unknown glucose delay in infants we correlated RG with CGMS values at 5.10.15.20.25 and 30 minutes following RG

Results We measured 264 RG in 20 infants and used 97 (37\%) for CGMS-calibration. The strongest correlation were at $20 \mathrm{~min}$ with $\mathrm{r}=0.709$ and corresponding MARD (mean absolute relative deviation) of $13.28 \%$. Plotted in Clark Error Grid showed that $94.5 \%$ of the pairs were in zone $\mathrm{A}$ and $\mathrm{B}$.

Conclusion Based on our findings it seems that CGMS is a reliable and feasible method for testing and following blood glucose levels in infants. The use of CGMS could reduce the amount of painful RGmeasurements to $37 \%$ and at the same time it increases the patient's safety through a much better glucose control.

The subcutaneous glucose delay in infants is approximately 20 minutes, which was not published before.

\section{EVALUATING VARIATION IN COLONIZATION FROM DIFFERENT PARTS OF UMBILICAL CORD AMONG NEONATES IN HOSPITAL SETTING IN DELHI AND PEMBA TANZANIA}

doi:10.1136/archdischild-2012-302724.1408

'S Gupta, ${ }^{2} \mathrm{U}$ Dhingra, $1 \mathrm{P}$ Dhingra, ${ }^{3} \mathrm{~A}$ Dutta, 'S Madhesiya, ${ }^{2} \mathrm{R}$ Black, ${ }^{3} \mathrm{SM}$ Ali, ${ }^{3} \mathrm{~S}$ Ame, ${ }^{3}$ S Deb, ${ }^{1}$ A Ahmed, ${ }^{2} S$ Sazawal. ${ }^{1}$ Center for Public Health Kinetics, New Delhi, India; International Health, Johns Hopkins Bloomberg School of Public Health, Baltimore, MD, USA; 'Public Health Laboratory-IdC, Chake Chake Pemba, Zanzibar, Tanzania

Background Umbilical cord is a potential portal of entry for invasive bacteria causing neonatal sepsis and death from serious infections. Studies have used a single swab covering tip, stump and base region for identifying umbilical cord colonization. Information for bacteriological profile of the cord evaluating variation from tip, stump and base is lacking. As a pilot for a large randomized controlled trial of Chlorhexidine intervention the present study aimed to evaluate the variation in colonization at three sites of cord tip/ stump/base.

Methods Newborns enrolled from hospitals in Delhi $(\mathrm{n}=56)$ and Pemba ( $n=68)$, three swabs were collected one each from tip, stump and base of the cord. Swabs were sent to laboratory within 6 hours of collection for identification of pathogens.

Results Positivity for bacterial colonization at tip was lower than stump and base. Highest positivity for bacterial growth was found at base (Delhi 0hr-16\%; 24hrs-23\%; Pemba 0hr-20\%) followed by stump (Delhi 0hr-7\%; 24hrs-27\%; Pemba 0hr-11\%). Percentage of newborns with positivity at tip was lowest with $5-6 \%, 3 \%$ and $1.97 \%$ among Delhi 0hr, 24hrs and Pemba 0hr respectively. At 24hr, the bacterial colonization for stump and base combination increased from $5 \%$ (at baseline) to $21.4 \%$.

Conclusion With non-significant variation between the three sites for bacterial isolation, for clinical trials evaluating association of colonization with clinical outcomes taking two swabs (one from tip and other from stump and base of the cord) should be adequate. Association between bacterial isolation at each of two sites with clinical events and mortality needs investigation.

\section{OLFACTORY STIMULATION REDUCES APNEA REFRACTORY TO CAFFEINE AND THE USE OF DOXAPRAM IN PREMATURE NEWBORNS}

doi:10.1136/archdischild-2012-302724.1409

${ }^{1} \mathrm{~L}$ Marlier, ${ }^{2} \mathrm{C}$ Gaugler, ${ }^{3} \mathrm{D}$ Astruc, ${ }^{4} \mathrm{~J}$ Messer. ${ }^{1}$ Neurosciences, National Center of Scientific Research CNRS-LINC-IPB, Strasbourg; ${ }^{2}$ Pediatrics, Hôpitaux Civils de Colmar, Colmar; ${ }^{3}$ Neonatology; ${ }^{4}$ Neonataology, Hôpitaux Universitaires de Strasbourg, Strasbourg; France

Idiopathic apnea of prematurity remains a major clinical problem that requires treatment. For three decades, methylxanthines (caffeine and theophylline) have remained as the primary treatment choice. Several studies have shown the effectiveness of doxapram in reducing apnea refractory to methylxanthines, but numerous side effects have been reported, so that its use remains controversial. In this study, we examined a novel therapy in the form of olfactory stimulation in premature newborns suffering from apnea refractory to caffeine. Thirteen premature newborns born at 28 to 31 gestational weeks were exposed to a pleasant odor diffused during 24 hours in the incubator. A reduction of the number of apnea during the day with odorization compared to the day before (baseline) or the day after (recovery) occurred in all infants. In mean, the reduction reached $42 \%$. Particularly, the apnea associated with episodes of bradycardia and hypoxemia decreased strongly (49\%) and affected all the infants. Due to this reduction, no additional treatment with doxapram was necessary. Taken together, these data indicate that the introduction of a pleasant odor in the incubator is of therapeutic value in the treatment of apnea unresponsive to caffeine and give a new possibility to reduce the use of the controversial doxapram.

\section{THE RELATIONSHIP OF MATERNAL ETHNICITY WITH INCIDENCE OF SHOULDER DYSTOCIA IN NEWBORNS: STUDY FROM A LONDON UNIVERSITY HOSPITAL}

doi:10.1136/archdischild-2012-302724.1410

S Khan, J Daniels. North Middlesex University Hospital, North Central London Deanery, London, UK

Aims To evaluate maternal ethnicity as a factor reported to increase the risk of shoulder dystocia, and to evaluate its predictive value at a population level.

Methods We conducted a retrospective cohort study of 252 singleton, vertex vaginal deliveries with diagnosed shoulder dystocia born at North Middlesex University Hospital in London, UK. Maternal ethnicity was examined along with maternal characteristics, induction and length of labour, operative vaginal delivery, epidural utilisation, and birth weight. These variables were analysed as factors affecting incidence of shoulder dystocia and subsequent neonatal injury.

Results Among women who met study criteria, 252 (2.4\%) experienced a shoulder dystocia. Women of black ethnic background (African and Afro-Carribean) had the highest percetage of shoulder dystocia (43.8\%), compared with White background (39.5\%) and the lowest was amongst women of Asian background (16.6\%). Additionally, in the setting of a shoulder dystocia, a higher risk of brachial plexus injury was observed in neonates delivered by women of Black background taking in to account confounding factors such as birth weight, gestational diabetes and previous shoulder dystocia.

Conclusions Overall women of black background have an increased risk of shoulder dystocia and their neonates are more likely to experience birth injury subsequently. Induction of labour and infant birth weight greater than $4000 \mathrm{~g}$ have traditionally been identified as significant independent predictors of shoulder dystocia; however ethnicity should be taken into account when considering caesarean section as prophylaxis against shoulder dystocia and neonatal investigations for possible brachial plexus injuries post birth. 
1411 VALIDATING THE AGREEMENT BETWEEN NEWER INFRARED FOREHEAD THERMOMETRY WITH AXILLARY DIGITAL THERMOMETRY IN NEONATES

doi:10.1136/archdischild-2012-302724.1411

'AR Sethi, 'AS Nimbalkar, 'DV Patel, ${ }^{2}$ AG Phatak, ${ }^{1}$ '2 SM Nimbalkar. 'Department of Pediatrics, Pramukhswami Medical College; ${ }^{2}$ Central Research Services, Charutar Arogya Mandal, Anand, India

Background and Aims The traditional mercury thermometer has been replaced by the more "user friendly" digital thermometer. As accuracy is comparable with both instruments and mercury remains an environmental hazard they are no longer recommended. New non invasive method of measuring temperature may reduce infection rate as well as intangible pain and suffering of neonate.

Methods The body temperature of patients admitted in Neonatal Intensive Care Unit was measured using axillary digital thermometer as well as a handheld infrared non touch thermometer. Patients placed under radiant warmers were included. Temperature recordings were taken as required routinely for clinical care. Axillary temperature was recorded within 30 seconds and the forehead temperature within 5 seconds.

Results The body temperature measured by Axillary digital thermometer and forehead method do not agree well (95\% limits of agreement: $-4.2,2.2)$. A trend was observed suggesting that agreement depends on the magnitude of the temperature. The agreement slightly improved when patients in warmer were excluded $(95 \%$ limits of agreement: $-4.1,2.1)$ with similar trend. The best possible agreement was observed between warmer and axillary temperature but was not clinically acceptable (95\% limits of agreement: -0.99 , 2.36).

Conclusion Forehead temperature due to non touch may appear less disturbing to the neonate and also time saving for the nurse but they are misleading. The infrared technology needs further improvement before it can be used in our setting. Although advent of technology is tempting, a scientific validation of new technology under different settings is caveat before adapting it.

\section{TRANSCUTANEOUS BILIRUBINOMETERS USED WITHIN A STRUCTURED PATHWAY PREDICTS HIGH SERUM BILIRUBIN LEVELS IN HEALTHY TERM JAUNDICED NEONATES MONITORED AT HOME}

doi:10.1136/archdischild-2012-302724.1412

'JO Menakaya, 'S Davies, ${ }^{2} \mathrm{~N}$ Callender, Jaundice Early Treatment Study In Term Newborns (JETSET). 'Neonatal Paediatrics; ${ }^{2}$ Obstetrics \& Gynaecology, Hillingdon Hospital NHS Foundation Trust., London, UK

Background UK (NICE) guidelines recommend that bilirubin is monitored in all jaundiced babies. We implemented a home based integrated care pathway to monitor healthy term jaundiced neonates with transcutaneous bilirubinometers $\left(T_{c} B\right)$. Babies were readmitted to hospital for phototherapy at total serum bilirubin $(\mathrm{TsB}) \geq 340 \mu \mathrm{mol} / \mathrm{l}$. TcB $\leq 250 \mu \mathrm{mol} / 1$ correlates highly with TsB.

Aim To determine the $T c B$ values in term babies monitored at home that could predict TsB values $\geq 340 \mu \mathrm{mol} / 1$.

Methods Healthy jaundiced neonates were monitored at home using Bilichek ${ }^{\circledR}$ (Ver 6.12) bilirubinometer. Babies with $\mathrm{TcB}>$ $250 \mu \mathrm{mol} / \mathrm{l}$ had TsB measured using the Beckman Coulter timed endpoint diazo method within 4 hours to confirm result. We carried out statistical analysis of the paired samples to determine the TcB value with the best predictive value for $\mathrm{TsB} \geq 340 \mu \mathrm{mol} / 1$.

Results Eighty-three paired samples were analysed from 63 babies $6(7 \%)$ had TsB values of $\geq 340 \mu \mathrm{mol} / \mathrm{l}$. The Receiver Operating Characteristics (ROC) curve analysis suggested an area under the curve (AUC) of 0.9037 . TcB values $\geq 315 \mu \mathrm{mol} / 1$ predicted $\mathrm{Ts} B \geq 340$ $\mu \mathrm{mol} / \mathrm{l}$ with sensitivity of $0.83(0.36,1.00)$; specificity of $0.82(0.71$,
$0.90)$; positive predictive value of $0.26(0.09,0.51)$ and overall accuracy of $0.82(0.72,0.90)$. TcB values $\geq 303 \mu \mathrm{mol} / 1$ predictive ability had a sensitivity of $1.00(0.54,1.00)$; specificity of $0.71(0.60,0.81)$, positive predictive value of $0.21(0.08,0.41)$ and overall accuracy $0.73(0.63,0.83)$.

Conclusion Bilichek ${ }^{\circledR} \mathrm{TcB}$ of $303 \mu \mathrm{mol} / 1$ had higher sensitivity but lower specificity than TcB of $315 \mu \mathrm{mol} / \mathrm{l}$ for predicting TsB values $\geq 340 \mu \mathrm{mol} / 1$ in healthy term jaundiced neonates monitored at home.

\section{RELATION BETWEEN MATERNAL BLOOD LEVEL OF SOME METALS AND BIRTH WEIGHT USING LIBS}

doi:10.1136/archdischild-2012-302724.1413

\section{J Alsharnoubi. Cairo University, Cairo, Egypt}

Objective Aim of this study is to investigate the relation between intensities of some blood metals as $\mathrm{Fe}, \mathrm{Zn}, \mathrm{Pb}$, and $\mathrm{Cd}$, in both mothers and their newborn, and their effects on the newborn birth weight, height as well as head circumference using Laser Induced Breakdown spectroscopy LIBS as a non invasive technique.

Methods 34 pregnant women and their normal birth weight newborn (NBW, group I) were recruited and matched against 34 pregnant women and their low birth weight newborn (LBW, group II). Blood samples were collected from the umbilical cords of the newborns from both groups and venous blood samples were taken from their mothers after delivery. Samples were prepared then exposed to laser. We used the laser induced breakdown Spectroscopy (LIBS), to analyze the metal intensity of $(\mathrm{Cd}, \mathrm{Pb}, \mathrm{Zn}$, and $\mathrm{Fe})$, for each sample for both mother and her infant.

Results There were significant differences between both groups regarding $\mathrm{Cd}, \mathrm{Pb}, \mathrm{Zn}$, more in group I, while there were no significant differences in both groups in the mother and her newborn regarding to Fe. There were significant differences regarding $\mathrm{Cd}, \mathrm{Pb}$, $\mathrm{Zn}$, more in group I, while no significant differences in both groups in the mother and her newborn regarding to Fe.

Conclusion We found that there was significant negative correlation between birth weight and maternal blood intensity in both groups. We found positive correlation between maternal and newborn blood metals and a negative correlation between intensity of both maternal and infant blood metals regarding weight in both groups.

\section{INSIGHTS INTO NEONATAL ORAL FEEDING PATHOLOGY THROUGH RNA SEQUENCING OF SALIVARY SAMPLES}

doi:10.1136/archdischild-2012-302724.1414

1,2 JL Maron, ${ }^{3} \mathrm{KL}$ Bodi, ${ }^{3} \mathrm{KL}$ Johnson, ${ }^{2,4} \mathrm{DW}$ Bianchi. 'Pediatrics, Floating Hospital for Children, Tufts University; ${ }^{2}$ Mother Infant Research Institute at Tufts Medical Center; ${ }^{3}$ Tufts University School of Medicine: ${ }^{4}$ Pediatrics, Division of Genetics, Boston, MA, USA

Background and Aims To improve our understanding of newborn feeding pathophysiology at the molecular level, our laboratory studies transcripts in neonatal saliva. Previously, we used whole transcriptome microarrays. Here, we tested the hypothesis that sequencing of RNA would provide additional and more specific information.

Methods RNA was extracted and prepared for sequencing from salivary samples $(10 \mu \mathrm{L})$ collected from two term infants matched for post-conceptual age, gender and ethnicity who could and could not orally feed, respectively. Paired-end $100 \times 100$ base pair sequencing was performed on the Illumina HiSeq 2000. Sequence data were aligned against human reference genome GRCH37/hg19. Cuffdiff analysis identified differentially expressed genes, promoters, and splicing variants between subjects. Ingenuity Pathway Analysis was performed on statistically significantly differentially expressed genes. 